ding a Forum for Bioethies in U.S. Public Policy», Politics and Life Sciences, 12 (1993), pp. 205-219. Véase también el conjunto de contribuciones sobre biostica y polftica pública en el número de febrero de 1994 de Politics and Life Sciences, 13, pp. 77-101.

37. David Shapiro sugiere las siguientes coordenadas para clasificar los comités de ética:

$$
\begin{gathered}
\text { nacional - local } \\
\text { con estatuto jurídico - voluntario } \\
\text { centralizado - plural } \\
\text { regulador - consultivo }
\end{gathered}
$$

$$
\begin{aligned}
& \text { general - especifico } \\
& \text { profesional - público } \\
& \text { permanente - ad hoc }
\end{aligned}
$$

Véase D. Shapiro, *A Single National Voice for Bioethics: Reflections from Europes, Politics and the Life Sciences, 13 (1994), pp. 98-99.

38. K. Shrader-Frechette, *Technology Assessment as Applied Philosophy of Sciences, Science, Technology \& Humant Values, 33 (1980), pp. 3350; y A. Rip, aControversies as Informal Technology Assessment", Knowledge: Creation, Diffusion, Utilization, 8 (1986), pp. 346-371.

\title{
Tecnología y filosofía: más allá de los prejuicios epistemológicos y humanistas
}

\author{
MANUEL MEDINA \\ Universidad de Barcelona
}

En el índice de casi sesenta páginas de la Encyclopedia of Philosophy editada por Paul Edwards no figura ninguna entrada con el término "técnica» ni «tecnologia». Esta ausencia, en una obra bien conocida, ilustra claramente la notable ignorancia y marginación con la que la tradición filosófica ha tratado, hasta nuestros días, todo lo referente a las técnicas.

De hecho las relaciones entre técnica y filosafia quedaron ya sentenciadas en los mismos orígenes de la tradición filosófica. Platón y Aristóteles construyeron la división teórica entre techne y episteme y entre poiesis y praxis, es decir, entre las técnicas de producción material, por un lado, y el conocimiento teórico, la filosofía y las actividades no productivas, por otro. La separación teórica de la técnica respecto a la ciencia y las humanidades configura los prejuicios filosóficos que han acompañado la larga historia de la fi- losofía y sus relaciones con la técnica, llegando incluso a marcar la moderna filosofía de la tecnología y a enfrentar distintas corrientes dentro de la misma. La superación de dichos prejuicios, tanto en la filosofía de la tecnología como en la filosofía de la ciencia, pasa por la integración de ambas en una filosofía de la tecnociencia, dentro de los actuales estudios interdisciplinares de ciencia y tecnologia.

\section{Los prejuicios de la filosofia tradicional}

La originaria interpretación filosófica de la técnica partió del supuesto de que el conocimiento predicativo ( saber quép) representaba el conocimiento propiamente dicho, mientras que el conocimiento operativo ("saber cómon) quedaba relegado como mera empeiria. ${ }^{2}$ Por este camino se llegó al conocimiento térico (usaber por qué») o episteme como la forma supe- 
rior de conocimiento, centrada en la explicación teórica o deducción a partir de primeros principios. ${ }^{3}$ Para Platón (Gorgias) al igual que para Aristóteles (Metafísica y Etica a Nicomaco) las technai tenian que ver con logoi, es decir, con la verdad de enunciados. Según esta interpretación epistemológica, las technai correspondian a un conocimiento verdadero pero contingente, o sea, doxa que nunca podía alcanzar la categoría de conocimiento teórico, necesariamente verdadero e inmutable, representado por la episteme o ciencia. Los diversos tipos de técnicas se distinguian conforme a una gradación epistemologica según estuvieran más relacionadas con objetos simbólicos (de orden superior) como la aritmética, o con la producción de objetos materiales (de orden inferior) como la escultura.

En esta epistemologización de la técnica se asimilaba primeramente la misma al conocimiento predicativo para luego construir su subordinación esencial respecto a la episteme. Según esto, un artesano al producir un objeto material reproducía una forma que representaba una idea inaccesible a su propia técnica, puesto que ésta era ateorica. Sólo el térico tenía acceso a esa idea inmaterial $y$ era quien, como usuario, determinaba la forma que el artesano, a su servicio, tenía que reproducir. La techne era, pues, una aplicación subordinada de episteme.

Con estas interpretaciones filosoficas se funda el antiguo prejuicio epistemologico o teoricista, consistente en interpretar la técnica en términos epistemologicos para luego contraponer techne y episteme y desembocar, finalmente, en la descalificación epistemológica de las técnicas y en el primado de la teoría.

En la caracterización que da Aristoteles de techne en el capítulo cuarto del libro VI de su Ética a Nicómaco no sólo aparece el prejuicio episternológico (elaborado en el capítulo anterior), sino que incluye un segundo prejuicio filosófico. Según Aristóteles, toda tech-

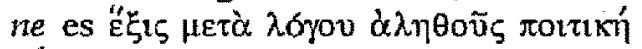
(Ética a Nicómaco 6.4.1140a11), traducido literalmente, «la capacidad de producir objetos materiales conforme con un logos verdadero". En esta definicion queda patente el prejuicio epis-

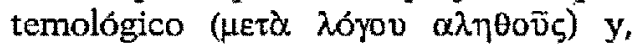
además, se identifica techne con la prodtucción de objetos materiales o poiesis. A lo largo de todo el capítulo cuarto Aristóteles repite machacona-

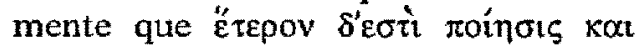
$\pi \rho \tilde{\alpha} \xi\llcorner$, , esto es, la poiesis (producción material) y la praxis (actividades no productivas propias del hombre libre) son cosas completamente distintas. Por tanto, hay que separar las técnicas productivas de las capacidades humanas "superiores", entre las que destacan las discursivas y teóricas (como la filosofía), las políticas y las de disfrute.

Ya Platón (Philebo) había separado el dominio de la producción material del de la actividad y el conocimiento de tipo político (como la educación o el gobierno) y lo habia asociado al ambito de las cosas materiales no-humanas. ${ }^{4} \mathrm{Y}$ Aristóteles está de acuerdo con él en separar la técnica de lo concerniente a los asuntos humanos, especialmente los políticos. ${ }^{5}$ Las teorias filosoficas de la techne junto con la interpretación epistemológica de la politica como conocimiento teorico conducen directamente, en la filosoffa platónica y aristotélica, a la descalificación politica de las technai y de los artesanos que las ejercen. En estas divisiones y contraposiciones entre el mundo de lo humano y el mundo de lo no-humano o técnica, entre los asuntos politicos, sociales y culturales y los artefactos materiales, se asienta el prejui- 
cio humanista de la filosofia con relación a la técnica.

Cuatro siglos más tarde encontramos el tándem de los prejuicios epistemológico y humanista funcionando claramente en las interpretaciones filosóficas de la ciencia y la técnica. En su obra Vidas Paralelas, Plutarco relata las grandes dificultades que tuvo el cónsul romano Marcelo para conquistar Siracusa debido a las invenciones de $\mathrm{Ar}$ químedes para defender la ciudad. En esta época las technai artesanales habían dado paso a tecnologías ingenieriles desarrolladas en conexión con tratados de mecánica teórica, entre los que destacan los del propio Arquímedes. La invención y desarrollo de este tipo de tecnologias, especialmente las relacionadas con la guerra, pasaron a ser objeto de gran interés y promoción politica, sobre todo a partir de Alejandro Magno.

$\mathrm{Al}$ explicar las invenciones tecnologicas de Arquímedes asociadas con artefactos materiales de tipo mecánico, Plutarco las presenta como aplicaciones de sus grandes conocimientos científicos. Así, el polipasto que da lugar a la espectacular demostración de Arquímedes en Siracusa sería una aplicación de su teorema de la palanca:

Arquimedes escribió al rey Hierón, pariente y amigo suyo, que con una fuerza dada se podía mover cualquier peso; y se dice que, alentado por la fuerza de la demostración, declaró que si hubiera otra tierтa y se pudiera trasladar a ella, podria mover ésta. Hierón quedó maravillado y le pidió que aplicara su teoría y le mostrara un gran peso movido por una pequeña fuerza. Entonces Arquimedes escogió un barco de carga de tres velas de la flota real que habian sacado a tierra muchos hombres con gmondes esfuerzos $y$, después de poner a bordo gran número de pasajeros y la carga habitual, se sentó a distancia, y sin gran esfuerzo, sólo accionando con su mano un polipasto [sistema de poleas compuestas]. lo movió hacia sí suave y uniformemente como si estuviera deslizándose sobre el mar. El rey, estupefacto y comprendiendo el poder de la técnica, encargo a Arquímedes que le construyera máquinas de defensa y ataque para todo tipo de asedio.? $^{7}$

Pese a reconocer la importancia social y política de este tipo de técnicas, Plutarco insiste en su versión platónica de la figura de Arquímedes, en la que se mantiene la separación entre la vil y desdeñable técnica material y la sublime excelencia de la teoria.

En cuanto a Arquímedes, poseia un espiritu tan elevado y profundo y una riqueza tal de tcorias cientificas que, aunque sus inventos le habian ganado renombre $y$ fama de una inteligencia sobrehumana, no consintió en dejar ningún tratado sobre esta materia, sino que considerando las prácticas mecánicas y en general todas las técnicas relativas a las necesidades de la vida como viles y manuales, dedicó todos sus esfuerzos sólo a aquellos estudios cuya belleza y excelencia no se mezclan con las necesidades materiales. Estos estudios no se pueden comparar con los otros y en ellos el objeto de estudio rivaliza con la demostración, aquél proporcionando la grandeza y la belleza y ésta una precisión y un poder insuperables. ${ }^{8}$

Como autoridad legitimadora de la gran división entre las técnicas y artefactos materiales, por un lado; y la teoría $\mathrm{y}$ las actividades no productivas, por otro, Plutarco señala a Platón. El principal responsable, según él, de que la mecánica fuera desestimada como objeto de interés filosófico.

Pues la técnica mecánica, ahora tan apreciada y celebrada, habia sido cjercida anteriomente por Eudoxo y Arquitas, quienes adomaron la geometría con sutilezas e ilustraron con ejemplos mecánicos intuitivos problemas geométricos que no podian 
resolverse teóricamente. Asi, para resolver el problema de encontrar dos segmentos proporcionales entre si, requisito necesario para muchas figuras geométricas, habían echado mano de recursos mecánicos empleando mesolabios derivados de curvas y secciones cónicas. Pero Platón se enfureció con esto y los condenó como comuptores y destructores de la pura excelencia de la geometría, que de esta forma sc apartaba de las cosas incorporeas del pensamiento abstracto y descendía a las cosas sensibles haciendo uso de objetos propios de pesados y viles oficios manuales. Por esta razón la mecánica se separó completamente de la geometria y fue despreciada durante mucho tiempo por la filosofía, pasando a ser considerada como una técnica militar."

Así pues no es de extrañar que los prejuicios filosóficos originarios consiguieran mantener la relegación de la técnica a lo largo de la historia de la filosofía. Habrá que esperar al siglo XIX para que surja un interés teórico por la técnica y hasta el último tercio del siglo $X X$ para que empiece a institucionalizarse, paulatinamente, un nuevo campo académico dedicado a la filosolía de la tecnologia.

\section{La moderna filosofía de la tecnologia}

Karl Marx fue el primer teórico que atribuyó a la técnica un papel central en la construcción de sus teorías. $\mathrm{Al}$ "poner a Hegel sobre sus pies" Marx reinvirtió también, de algún modo, el prejuicio del humanismo filosófico acerca de la técnica, emplazándola como motor de la emancipación humana en su teoría del desarrollo histórico. Según esta teoría, el desarrollo de los medios de producción, determinado por las innovaciones técnicas, es el que configura los cambios en las estructuras socio-políticas e ideologicas. Su "materialismo» o humanismo materialista consiste, precisamente, en conceder a las técnicas materiales la primacía sobre el desarrollo político y cultural. Marx centró su análisis en la moderna tecnología industrial y reconocio anticipadamente las tendencias hacia la sistematización tecnológica y la automatización de la producción.

Sin embargo, el primer autor en acuñar la expresión "filosofía de la técnica» fue el geógrafo antimarxista Ernst Kapp. En su obra Grundlinien einer Philosophie der Technik, publicada en 1877, desarrollo una interpretación de las invenciones e instrumentos técnicos como proyecciones de los órganos humanos. Desde una perspectiva optimista, Kapp intentó una revalorización neohegeliana de la técnica como propulsora del desarrollo cultural, moral e intelectual.

En esta misma línea de valoración positiva, el ingeniero Friedrich Dessauer repite el título Philosophie der Technik en una obra publicada en 1927. Para Dessauer las invenciones técnicas consisten en aproximaciones a la solución ideal, platónicamente preestablecida, de problemas técnicos. El hombre sólo actualiza formas técnicas existentes ya en potencia y, al hacerlo, obra como instrumento de Dios para continuar la creación. En la filosofía de Dessauer reencontramos el intento de revalorizar la técnica y el estamento ingenieril, que el autor escala hasta llegar a la rehabilitación teológica.

Iosé Ortega y Gasset es el primer filósofo profesional que dedica expresamente un estudio a la técnica. Su $\mathrm{Me}$ ditación de la técnica se publica en forma de libro en 1939, pero recoge los escritos para un curso impartido en 1933 en la universidad de verano de Santander, que habian sido ya publicados en forma de articulos por $\mathrm{La} \mathrm{Na-}$ ción de Buenos Aires en 1935. La obra 
empieza anticipando que «uno de los temas que en los próximos años se va a debatir con mayor brío es el sentido, ventajas, daños y límites de la técnica» ${ }^{10}$ La interpretación orteguiana de la técnica no es trascendental, sino más bien existencial. Para Ortega el hombre es un ser técnico determinado biológica pero no culturalmente, cel ser para el cual lo superfluo es necesarios. La técnica representa ala creación de posibilidades siempre nuevas que no hay en la naturaleza del hombren, posibilitando asi la realización de diferentes eplanes vitales».

A diferencia de Ortega, cuyos escritos sobre la técnica apenas encontraron eco ni entre sus propios seguidores, otro filosofo profesional, Martin Heiddeger, habría de ejercer con su opisculo La pregunta por la técnica, editado en 1954, un notable intlujo en la corriente fenomenológica y humanista de la filosofia de la tecnologia norteamericana. Su interpretación filosófica de la técnica y de la relación de ésta con la ciencia se centra en el carácter esencialmente tecnológico de la física moderna, constatación a la que debió llegar a través de la lectura de La imagen de la Naturaleza en la física actual de Heisenberg, al que él mismo menciona en su obra:

Se dice que la técnica moderna es incomparablemente diferente de todas las anteriores porque se basa en la moderna ciencia natural exacta. Entretanto se ha reconocido con más claridad que también la inversa es cierta: la física moderna, en cuanto experimental, depende del aparataje técnico y del progreso de la construcción de aparatos. $\mathrm{La}$ constatación de esta relación mutua entre técnica y física es correcta."

En la terminología heiddegeriana, la técnica moderna se interpreta como una imposición a la naturaleza para provocar su desocultamiento como un complejo calculable de fuerzas. ${ }^{2}$ En términos menos esotéricos se podría decir que los procesos energéticos provocados, controlados y reproducidos mediante los artefactos creados por la física constituyen la naturaleza que se arevela» en la ciencia. Dadas las características de la física y de la tecnica modernas no se puede afirmar, a juicio de Heiddeger, que la técnica sea ciencia aplicada, sino más bien lo contrario:

Por el hecho de que la esencia de la técnica moderna se basa en la imposición, esa técnica ha de utilizar la ciencia natural exacta. De ahí nace la apariencia engañosa de que la técnica moderna es ciencia natural aplicada. Esta apariencia se puede mantener en tanto no se indaga suficientemente ni el origen esencial de la ciencia moderna ni todavía menos la esencia de la técnica moderna. ${ }^{13}$

Hasta aquí Heiddeger interpreta en términos de su propia filosofía lo que el físico Werner Heisenberg y otros teóricos de la física, como Hugo Dingler,"14 habian ya constatado. Lo más original de su filosofía de la técnica son las conclusiones y valoraciones negativas a las que llega en la línea de los prejuicios del humanismo filosófico tradicional. Según Heiddeger, el carácter de la técnica y la ciencia moderna representa un aextremo peligro, no sólo para la esencia del hombre, sino para todo desocultar en cuanto talm.15 Y "la verdadera amenaza ya ha afectado al hombre en su esencia. El dominio de la imposición amenaza con la posibilidad de que al hombre le pudiera ser negado el acceder a un desocultar más originario y de este modo experimentar el aliento de una verdad más inicial"." 6 .

Estas y otras heterogéneas protofilo- 
soffas de la técnica diffcilmente hubieran llegado por sí mismas a configurar un territorio filosófico propio. De hecho, han sido los incansables esfuerzos de algunos profesionales de la filosofía los que han ido logrando establecer lentamente, en algunos parses, una filosofia de la tecnologia como disciplina por derecho propio. Sobre todo a partir de los años setenta, filosofos provenientes de diversas corrientes llegaron a unirse en EE.UU. y Alemania en el empeño común de crear las bases institucionales de una nueva rama de la filosofía, al igual que se habia conseguido para la filosofía de la ciencia en la primera mitad del siglo.

La primera reunión académica en que se planteó la filosofía de la tecnología como una meta concreta tuvo lugar a la sombra del VIII Congreso Anual de la Society for the History of Technology (SHOT), celebrado en San Francisco en 1965. En dicho congreso se organizó un simposio con el nombre de "Toward a Philosophy of Technology" en el que intervinieron, entre otros, Joseph Agassi, Mario Bunge, Lewis Mumford y Henryk Skolimovski. Las actas del simposio aparecieron al año siguiente en la revista de la SHOT Technology and Culture. Por esta misma época el tema de la filosofia de la tecnología resonaba también dentro del colectivo filosófico internacional. En el marco del XTV Congreso Mundial de Filosofía, que se celebró en Viena en 1968, tuvo lugar un coloquio especial dedicado a "Cibernética y Filosofia de la Tecnología, al que se presentaron numerosas contribuciones.

Pero habría que esperar a la década de los setenta para que empezaran a proliferar obras filosóficas dedicadas a la tecnología y, sobre todo, para que cuajaran los intentos de institucionalización en los EE.UU. Paul Durbin, a quien se ha llamado con razón el "pa- drino" de la filosofía de la tecnología norteamericana, organiz6 en $1975 \mathrm{y}$ 1977 congresos sobre «Filosofía y Tecnologran en la Universidad de Delaware. A partir de aqui cristalizaron los soportes institucionales de la filosofía de la tecnologra en Norteamérica. La Philosophy and Technology Newsletter, la coleccion Research in Philosophy and Technology, que más tarde se llamará simplemente Philosophy and Technology y será la publicación oficial de la Society for Philosophy and Technology (SPT). Esta asociación, tras un periodo de funcionamiento más o menos informal, se organiza formalmente en 1983, casi exactamente cincuenta años después de que se fundara la Philosophy of Science Association (PSA).

A principios de la década de los ochenta, el filosofo alemán Friedrich Rapp, quien habia editado Contributions to a Philosophy of Technology en 1974, se pone en contacto con Paul Durbin para organizar un congreso conjunto de los grupos norteamericano y alemán que estaban promocionando la filosofía de la tecnología en sus respectivos paises. Este congreso, que tiene lugar en 1981 en Bad Homburg, Alemania, y cuyas actas editarán conjuntamente Durbin y Rapp en 1983 con el título Philosophy and Technology, inaugura la serie de congresos bienales de la Society for Philosophy and Technology que se han venido celebrando regularmente a partir de entonces.

Desde hace ahora más de veinte años, los promotores de la filosofía de la tecnología han ido acotando un nuevo territorio que es muy difícil de cartografiar, debido a la heterogeneidad de los tratamientos valoraciones de la tecnología que se engloban. No obstante, se han esbozado diversos mapas en forma dc introducciones a la filosofía de la tecnología que, de hecho, co- 
rresponden a las distintas concepciones filosoficas y perspectivas nacionales (básicamente alemana, francesa y norteamericana). ${ }^{17}$

Algunas tendencias de la filosofia de la tecnología se enmarcan explícitamente en determinadas corrientes de la filosoffa tradicional. Así, en EE.UU. se da una corriente fenomenológica que entronca con la obra de Heiddeger y que se autoconsidera como un desarrollo de sus ideas. ${ }^{18}$ También se podría hablar de una filosofía politica de la tecnología, en la que cabría distinguir tendencias con influencias marxistas, de Herbert Marcuse, Jürgen Habermas..., y otras tendencias con distintas influencias como las de la tilosofia de Dewey. ${ }^{19}$ Pero, en general, no se pueden clasificar planteamientos teoricos muy definidos sino más bien afinidades conceptuales y valorativas generales.

A mi entender, lo más destacable del nuevo campo de la filosofia de la tecnología es su polarización en dos grandes corrientes contrapuestas que representan y desarrollan, en la actualidad, los prejuicios tradicionales de la filosofia respecto a la técnica. En consonancia con dichos prejuicios, podemos llamar a estas corrientes fitosofia humanista y filosofia analítica o epistemologica de la tecnología.

\section{Filosofia humanista y filosofía analitica de la tecnologia}

En la formación de la filosofía humanista de la tecnología en Norteamérica han tenido una notable influencia representantes de la filosofia continental como Martin Heidegger y Jacques Ellul. Este último, influenciado el mismo por Heidegger y el teólogo Karl Barth, publicó en 1954 su primera obra, La technique ou lenjeu du siecle, en la línea pesimista heideggeriana $\mathrm{y}$ de valoración negativa de la técnica propia de la filosofía tradicional, que Goffi llama la stechnophobie éternelle». ${ }^{20}$ Para Ellul «el mundo técnico es el mundo de las cosas materiales; se compone de cosas materiales y con relación a ellas. Cuando la técnica muestra algún interés por el hombre, lo hace para convertirlo en un objeto material"." Según el, "la técnica nunca puede engendrar libertad ${ }^{22}$ y el desarrollo imparable de la tecnología, que se rige en su teoria por una especie de lógica interna determinista, tiende a absorber la naturaleza y todas las formas de cultura humana. Su propuesta frente a los graves peligros que se derivan de la moderna confianza en la tecnología, son soluciones que trascienden la propia tecnología para basarse en la fe biblica. ${ }^{23}$

La corriente humanista tiene un peso notable dentro de la filosofía de la tecnología y marca, en mayor o menor medida, la obra de distintos filosofos. El autor que mejor contrasta con la filosofía analítica de la tecnología es Carl Mitcham, quien ha hecho de la filosofia humanista su estandarte en la defensa del primado de la propia filosofía frente a la tecnologia. Mitcham, uno de los principales promotores y cronista de la filosofía de la tecnología, contrapone la que él llama Humanities Philosophy of Technology ${ }^{24}$ a la Engineering Philosophy of Technology. Contrariamente a los wanálisis de la tecnología desde dentro" ${ }^{25}$ y a la "primacía conceptual de la fabricación de artefactos materiales" ${ }^{26}$ y su carácter "paradigmático para otros tipos de pensamiento y acción" ${ }^{27}$ características propias, según Mitcham, de la filosofía singenieril» de la tecnología (la tradición de Kapp y Dessauer), la filosofía humanista de la tecnologia ha de entenderse como wel intento de la religion, la poesía y la filosofía de que las 
perspectivas no tecnológicas o transtecnológicas influyan en la interpretación del significado de la tecnologían. ${ }^{28}$

En este programa filosofico, al contrario que en la filosofia «ingenieril», la pregunta por la tecnología "no está al servicio de ésta», sino que, en realidad, puede estar incluso dirigida "a frenar o interrumpir el desarrollo tecnológicon. ${ }^{29}$ La filosofía, que para Mitcham es una actividad claramente distinta de la tecnología, está llamada a salvar a nuestra época de la destrucción tecnológica de "muchas cosas que por otras razones se consideran verdaderas, buenas, bellas, justas o reales ${ }^{30}$ Para ello parte de la pregunta fundamental por lo humano, pregunta que «quizás no puede llegar a tener una respuesta definitivam. ${ }^{31} \mathrm{El}$ principio fundamental de la filosofía humanista de la tecnología es el primado de las humanidades (entre las que figura en lugar destacado la propia filosofia) sobre las tecnologias. ${ }^{32} \mathrm{De}$ dicho principio se deriva la prioridad de dicha filosofía respecto a la filosofía uingenieril» de la tecnologia. ${ }^{33}$

Ciertamente Mitcham ocupa una posición más bien extrema dentro del abigarrado campo de la filosofía de la tecnologia. Pero sus planteamientos filosóficos, como los de su inspirador Ellul, exhiben una serie de caracteristicas que, diversamente distribuidas y ponderadas, son compartidas por otros autores, configurando la corriente humanista en filosofía de la tecnología. Entre las tendencias más características de esta corriente destaca una actitud crítica respecto a la tecnología que puede ir desde la crítica cultural y social hasta posiciones que algunos criticos tachan de antitecnológicas. El tratamiento teórico que se hace de las cuestiones tecnológicas se basa, por lo general, en la especulación propia de la filosofia tradicional, llegándose has- ta planteamientos metafísicos y religiosos. Su foco de atención se centra, más que en teorías sistemáticas sobre la naturaleza de la tecnología, en los impactos de la misma y la valoración ética con predominio de los tonos negativos. Para hacer frente a las crisis tecnologicas dictaminadas por esta misma filosofía, se propugna, generalmente, un activismo militante en favor de soluciones «humanistas» por encima de planteamientos tecnologicos específicos.

Esta caracterización ideal-típica de la filosofía humanista contrasta con la que perfilaría la filosofía analítica de la tecnología. La actitud característica de la corriente analítica es protecnológica, pudiendo llegar su tecnofilia filosófica hasta la exaltación de la tecnologia como quintaesencia de la acción racional. En general se pretende un tratamiento descriptivo-teórico sistemático y riguroso, en el que se llega a recurrir ocasionalmente a la formalización. El objetivo principal de las elaboraciones teóricas tiende a centrarse en la investigación de la naturaleza misma de la tecnología. Implícitamente se da por supuesta una cierta neutralidad de los estudios filosóficos con relación a pronunciamientos sobre cuestiones valorativas. Según esta versión de la filosofía de la tecnología, la solución a los eventuales problemas planteados por las tecnologias es una cuestión que concierne a la propia investigación científica y tecnológica.

El primero en utilizar la expresión de filosofía analítica de la técnica fue Friedrich Rapp en una obra suya de 1978, que lleva ese mismo título. El autor relaciona expresamente su planteamiento filosófico con la filosofía analítica de la ciencia. Al igual que esta última se centra en el análisis de las estructuras teoricas, la filosofía analítica de la técnica tiene por objeto 
"el análisis estructural de la acción dirigida a un fin concreto", para "tratar de comprender el estatus metodológico y -además gnoseológico- del actuar técnicon. ${ }^{34} \mathrm{El}$ planteamiento analitico de la filosofía de la técnica "no intenta formular juicios de antemano acerca de cuestiones de contenidon ${ }^{35}$ y se reafirma, por lo demás, en el principio de la uneutralidad de los medios técni$\cos m .36$

Rapp forma parte de un grupo de filósofos alemanes surgido en torno al Verein Deutscher Ingenieure (VDI), ${ }^{37}$ al que también pertenecen, entre otros, Hans Lenk, Günter Ropohl y Walther Zimmerli. Desde los años setenta, este grupo ha impulsado de forma muy significativa la filosofía de la tecnología en Alemania y ha logrado establecerla en los curricula de institutos y universidades politécnicas. La filosofía alemana de la tecnología tiene un marca. do carácter ingenieril al haberse desarrollado con el apoyo del influyente VDI y dentro de la tradición filosófica de Kapp y Dessauer..$^{38}$ Para Rapp, cla capacidad de rendimiento de la técnica moderna" no sólo "se basa en la aplicación de los principios de las ciencias naturales" sino también "de los conocimientos de las ciencias de ingenieríam. ${ }^{39}$ Estos últimos tienen en común con los primeros «el método empírico y la formación matemática de teorías" ${ }^{\text {*0 }}$ Es decir, según esta filosofía, la tecnología es ciencía natural y ciencia ingenieril aplicadas.

En Norteamérica, Mario Bunge es el autor que más sistemáticamente ha transportado los planteamientos de la filosofía analítica de la ciencia a la filosofía de la tecnología y que más claramente se contrapone a la filosofía humanista de la tecnología. ${ }^{41}$ Así como la filosofia tradicional de la ciencia se centra en el análisis del método, las leyes y las teorías cientificas, las cuestio- nes centrales de la filosoffa de la tecnología serían, según Bunge, a) la existencia de un método tecnológico paralelo al método científico, b) la existencia de leyes tecnológicas $\mathrm{y}$, en su caso, su diferencia respecto a las leyes cientificas, y c) la peculiaridad de las reglas tecnológicas en relación con las reglas científicas. ${ }^{42}$

Para aplicar el tipo de análisis propio de la filosofía de la ciencia, había que encontrar en el campo de la tecnología (identificada usualmente con artefactos y procedimientos) formulaciones lingüísticas que, de alguna forma, fueran equivalentes a los sistemas conceptuales y teoricos con los que se identifica analíticamente a la ciencia. La interpretación de la tecnología como ciencia aplicada resuelve este problema. ${ }^{43}$ En primer lugar, se descartan como no pertenecientes a la tecnologia el saber práctico o saber-cómoproceder, por tratarse de umera habilidad o capacidad en vez de conocimiento conceptual", , y las "recetas de las artes y oficios y de la producción». ${ }^{45}$ Para Bunge, lo que caracteriza la tecnología y constituye el estudio central de la filosofia de la tecnología son las reglas tecnológicas o normas fundamentales de la acción. ${ }^{46}$

Según esto, las reglas tecnologicas son einstrucciones para realizar un número finito de actos en un orden dado y con un objetivo determinado" y "pueden simbolizarse por una cadena de signos». ${ }^{47}$ Son normas que windican cómo debe uno proceder para conseguir un fin determinado* y están además basadas een un conjunto de fórmulas de leyes capaces de dar razón de su efectividad $\gg .43$ Bunge destila como quintaesencia de la tecnologia las reglas tecnológicas, entendidas como formulaciones lingüísticas que representan teóricamente el saber tecnológico. De esta forma se llega a enunciados 
teóricos susceptibles de análisis lógico e incluso formal.

La condición de que las reglas tecnológicas han de estar fundamentadas en leyes científicas, establece el nexo directo entre la filosofía de la tecnología y la filosofía analítica de la ciencia. Dado que el modo analítico de fundamentar se basa en la derivación lógica y que en buena lógica asertoria no se pueden derivar reglas tecnológicas (enunciados normativos) de leyes científicas (enunciados nomológicos), Bunge introduce lo que el llama enunciados nomopragmáticos, a guisa de una especie de equivalentes asertorios de las reglas, que permiten la derivación en cuestión. ${ }^{49}$ De este modo, la filosofía de la tecnología queda subordinada a la filosofía de la ciencia en lo que podría llamarse una filosofia de la ciencia de la tecnología, no sólo en cuanto a la metodología analítica, sino también con relación a los mismos contenidos. La filosofía analítica transfiere la interpretación nomologica de la ciencia a la tecnologia, para acabar fundamentando la efectividad tecnológica en la verdad cientifica.

Esta filosofía tiene importantes consecuencias para el método tecnológico y la valoración de tecnologías. De acuerdo con la misma, la investigación tecnológica ha de epartir de teorías para llegar a reglas ${ }^{50}$ intentando «transformar algunas fórmulas legaliformes en reglas tecnológicas efectivas"." De hecho, en la visión de Bunge, «los cientificos y los tecnologos ela* boran reglas sobre la base de las teorías cientificas y los técnicos aplican dichas reglas".52 Es decir, la tecnologia serfa ciencia aplicada y la ingenieria, a su vez, aplicaría la tecnología.

Dada por supuesta la superioridad del conocimiento científico por encima de toda otra forma de conocimiento, es fácil pasar de las presuntas relacio- nes entre ciencia pura y tecnología a la excelencia y superioridad de las modernas tecnologías frente a las técnicas tradicionales de wlas artes y oficios precientíficos". ${ }^{53}$ Para la filosofía analitica, las reglas tecnologicas "prescriben el curso de la acción práctica optima ${ }^{54}$ y los actos guiados por la tecnología «pueden considerarse máximamente racionales». ${ }^{53}$

\section{Los modernos prejuicios filosoficos}

Entre la filosofía humanista y la filosofía analítica de la tecnología existe, pues, un claro enfrentamiento tanto en la valoración de la tecnología modema como respecto al papel de la filosofía. ${ }^{56}$ La revalorización o cl rechazo de la tecnología dependen de las diferentes interpretaciones de la misma, según se priman, respectivamente, las nuevas versiones del prejuicio epistemológico o humanista.

En la interpretación de la filosofía analítica, que se entiende a sí misma como una filosofia moderna de tipo "científicon, la tecnología es una forma derivada de conocimiento científico $y$, por tanto, participa de la excelencia del mismo. En cambio, para la filosofía humanista, heredera de la filosofía tradicional, ${ }^{57}$ la tecnología representa el moderno dominio de los artefactos materiales opuesto a las actividades y valores humanos superiores. Ambas concepciones coinciden, sin embargo, en la defensa de la subordinación de la tecnología: con relación a la ciencia, según la filosofia analítica, y respecto a la propia filosofía y las humanidades, según la filosofía humanista.

En la versión moderna del prejuicio humanista reencontramos la antigua separación platónico-aristotélica cntre el mundo de los asuntos humanos y el mundo técnico de los artefactos materiales. Dentro de esta tradición, Mit- 
cham define la tecnologia como "the making and using of artifacts", para puntualizar a renglón seguido: "human making, in turn, can be broadly distinguished from human doing - for example, political, moral, religious, and related activitiess.58 Además habría que excluir de la tecnología las artes, el lenguaje ${ }^{5 y} y$, por descontado la misma filosofía. ${ }^{60}$ Dado que la tecnología se entiende como esencialmente material, ${ }^{61}$ se supone que todos los asuntos humanos que se le contrapo. nen tienen un carácter "inmaterial" ajeno a lo artefactual. La preeminencia de la tecnología en nuestra cultura por encima de esas facultades «espirituales» pone en peligro, según denuncia insistentemente esta filosofía, los valores superiores humanos. Se trataría, pues, de invertir la preeminencia en cuestión restableciendo la subordinación de la tecnología moderna frente a la filosofía y todas las demás "humanidades". Conforme a esta doctrina del humanismo extra-tecnológico, las soluciones a los problemas tecnológicas trascienden el propio ámbito de la tecnologia.

Del lado analítico, la definición fundamental de tecnología como ciencia aplicada revive la vieja caracterización aristotélica de la techne como episteme aplicada. Sólo que la ciencia moderna ha ocupado el lugar de la episteme clásica y, al mismo tiempo, la condición de que la techne habra de conformarse a un logos verdadero que se ha traducido por la necesaria conformidad de la tecnología con las leyes cientifficas. Sin embargo, la moderna asimilación epistemológica de la tecnología no comporta ninguna equiparacion de ciencia y tecnología. La ciencia pura, según la filosofía analítica, es una empresa esencialmente intelectual de investigación teórica que debe deslindarse claramente de la tecnología. Cual- quier intento de subordinar la investigación científica a objetivos tecnologicos pondría, catastróficamente, en peligro su misma esencia. Por el contrario, es la tecnología la que debe permanecer subordinada a la creatividad científica como a su motor de desarroHo, si quiere conservar la excelencia por encirna de cualquier otro tipo de conocimiento técnico. Este principio de subordinación de la tecnología a la ciencia apuntala la subordinación metodológica de la filosofía de la tecnología a la filosofía analítica de la ciencia, de la que la primera sería algo así como una aplicación, ${ }^{62}$

Conclusión. El giro tecnológico: más alla de los prejuicios epistemológicos y humanistas acerca de la tecnología

Las doctrinas subordinativas tanto cpistemológicas como humanistas reflejan, en el fondo, las predilecciones de los filósofos por actividades consideradas como más afines a las suyas propias, ${ }^{63}$ justificando así, de paso, la pretensión de preeminencia de sus respectivas especialidades. En todo caso, está bastante claro que los viejos prejuicios tradicionales acerca de la técnica perviven incluso en la moderna filosoffa de la tecnologia. A mi modo de ver, la filosofía de la tecnología más libre de prejuicios tradicionales se encuentra en el campo de los nuevos estudios de ciencia y tecnología, donde se puede hablar de un giro tecrologico que invierte los antiguos presupuestos sobre las relaciones entre tecnologia, ciencia y sociedad.

Dicho giro tiene antecedentes en algunos heterodoxos de la tradición filosófica. Sin retrotraerse demasiado y quedándose en el ámbito general de la filosofía del siglo xx hay que destacar a John Dewey. Para Dewey la tecnología no se circunscribe a los artefactos 
materiales sino que puede considerarse como el conjunto de todas las capacidades humanas, incluyendo el lenguaje, la logica, las mismas ciencia y filosofía, así como las formas de organizacion social y política. ${ }^{64} \mathrm{La}$ ciencia constituye una forma especializada de práctica ${ }^{65}$ y es "una rama» y "un modo de tecnologian," no siendo los avances cientificos otra cosa que avances tecnológicos. ${ }^{67} \mathrm{De}$ todo ello se deriva que la comprensión de la tecnología es fundamental para comprender la ciencia y no a la inversa, como pretende la filosofía analítica.

Dentro ya del campo de la filosofía de la ciencia, hay que reconocer a la filosofía constructiva un lugar pionero en el estudio sistemático del carácter tecnologico de la ciencia y de su concepción como práctica y cultura. Esta filosofia, inspirada originariamente en los trabajos de Dingler, se empezo a desarrollar a partir de los años sesenta en Alemania. Paul Lorenzen ha sido el principal impulsor de la teoría constructiva sistemática de las ciencias. Su obra ha dado lugar a una importante corriente filosófica ${ }^{68}$ que anticipó en más de un decenio las tendencias constructivas en sociología del conocimiento científico.

Para Lorenzen la ciencia moderna use muestra como un producto de nuestra cultura técnica: se basa en una práctica precientifica exitosa". ${ }^{69}$. El reconocimiento de esta idea se ha ido abriendo camino lentamente en nuestros días:

En tcoria de la ciencia se empieza a reconocer lentamente en nuestro siglo con el llamado giro pragmático que toda ciencia (toda teoria) sólo puede comprenderse sobre la base de una técnica - ya parcialmente- exitosa. Las teorías son instrumentos lingäísticos en apoyo de una práctica ya en marcha. $^{70}$
La tarea de la filosofia constructiva de la ciencia empieza, precisamente, por conceptualizar y formular teóricamente esos procedimientos técnicos (constructivos) previos, para llegar a la comprension de las ciencias como sofisticadas prácticas tecnológicas que se han desarrollado con la ayuda de teorías. Desde sus mismos inicios, la teoría constructiva de la ciencia se ha centrado en la reconstrucción sistemática de disciplinas cientificas especificas como la matemática (desde la aritmética al análisis), la lógica, la geometría o la física. La matemática y la lógica constructivas se entienden como teorias del operar con símbolos (lo que puede llamarse técnicas simbolicas) en la práctica del cálculo o la argumentación. Geometria y física, en cambio, pertenecen a las "ciencias técnicas», en el sentido de técnicas materiales.

Así, por ejemplo, la geometría (teórica) euclidiana es una «ciencia fundamental" (Grundlagenwissenschaft) de nuestra tecnologia, por ser una condición para la reproducibilidad de las mediciones." Pero, a su vez, tiene un fundamento técnico. Este se basa sen una práctica que pertenece, desde hace al menos 5,000 años, a las técnicas elementales de nuestra cultura". ${ }^{72}$ Dichas técnicas de la construcción (material) de formas geométricas (superficies planas, esféricas...) y de cálculo geométrico precientifico -que alcanzaron un alto desarrollo en las antiguas culturas egipcias y babilónicas - constituyen la geometría técnica. La conceptualización teórica de tales operaciones técnicas de construcción geométrica, que recibe el nombre de Protogeometria, permite reconstruir el fundamento técnico de los conceptos fundamentales de la geometría térica. La teorización protogeométrica es un ejemplo claro de filosofía de la técnica implicada en las ciencias, que, en la filosofia 
constructiva, precede siempre a la interpretacion de las teorias científicas como construcciones teóricas. La filosofía de la técnica es, pues, una parte integrante fundamental de la filosofía constructiva de la ciencia.

En el caso de la física en general, los fundamentos técnicos (objeto de la llamada Protofisica) no sólo incluyen las técnicas de construcción y uso de los instrumentos de medición (esto es, además de la geometría técnica, la cronometría e hilometría técnicas para la medición de tiempos y masas) sino también la exuberante tecnología de los aparatos de reproducción y control de los efectos y procesos en los laboratorios. ${ }^{73}$ Los estudios constructivos han contribuido a poner de manifiesto la constitución esencialmente tecnológica de la física moderna, llegando a la conclusión de que más que de una presunta ciencia de la naturaleza, se trata fundamentalmente de tecnología, ${ }^{44}$ La filosofia constructiva reinvierte por completo los prejuicios analíticos acerca de las relaciones entre ciencia y tecnología, para entender la ciencia, más bien, como atecnolagía aplicada" ${ }^{75}$ Con ello queda reinvertido también el papel subordinado que la filosofia analítica asignaba a la filosofía de la tecnologia. En su planteamiento constructivo, filosoffa de la ciencia y filosofia de la tecnologia quedan integradas en lo que se podría caracterizar como filosofía de la tecnologia de la ciencia.76

Desde su institucionalización académica en los años sesenta, la historia de la tecnología ha contribuido a desmontar la tesis de la tecnología como ciencia aplicada, defendida también por algunos historiadores de la ciencia en contra de los estudios originarios de historia social de la ciencia. Pero el giro tecnológico es patente asimismo en la evolución más reciente de la misma historia de la ciencia. A partir de la década de los ochenta, han proliferado los estudios históricos que insisten en el papel central de los instrumentos y las tecnologías experimentales, producidas en los laboratorios, para el desarrollo de la ciencia. ${ }^{77}$ Así, Derek DeSo* lla Price ha puesto en primera línea el carácter tecnológico de la investigación científica moderna y la importancia decisiva de la innovación tecnológica de instrumentalidades para el cambio científico. Price sugiere, de hecho, una revisión tecnológica de las inexplicadas revoluciones kuhnianas. Estas vienen dadas por los cambios tecnologicos producidos en la investigación cientifica. ${ }^{78}$

Incluso desde la misma filosofía estándar de la ciencia han surgido autores como Ian Hacking, que se ha desmarcado de los prejuicios analíticos para reconocer que la ciencia no puede reducirse a las teorías científicas (representing), sino que la práctica experimental es esencialmente un modo de intervención tecnológica (intervening). ${ }^{79}$ Hacking propone abandonar la "moda" analítica de "don't talk about things, talk about the way we talk about things ${ }^{80}$ y dejar de hablar de enunciados observacionales para hablar de la actividad experimental. ${ }^{81} \mathrm{Se}-$ gún su filosofía, «las teorias de las ciencias de laboratorio no se comparan directamente con "el mundo"; persisten porque son verdaderas acerca de los fenómenos producidos o incluso creados por aparatos en el laboratorio y se miden mediante instrumentos que nosotros hemos construidon. 82

Los numerosos estudios de la sociología del conocimiento científico han sido decisivos para desmantelar definitivamente los prejuicioś epistemológicos, otrora dominantes desde la filosofía analítica de la ciencia, al poner de manifiesto el carácter constructivo y artefactual de la actividad científica. Sin 
embargo, en algunos planteamientos que sostienen la tesis de la construcción social radical de la ciencia y también de la tecnología, continúa presente el prejuicio humanista en una "modalidad sociologican. En este caso, lo humano no se identifica con lo filosófico, sino con lo social. Se da por supuesto que existe, por un lado, lo social "puro" (los actores humanos, las relaciones e interacciones sociales sin intervención de artefactos) y, al otro lado de la división, tendríamos los objetos (los no humanos sin conexiones sociales). El primado humanista consiste, en esta version, en primar unilateralmente la explicación sociologica pura (es decir, partiendo únicamente de causas sociales) del cambio científico y tecnológico. ${ }^{83} \mathrm{Al}$ presentar la ciencia y la tecnología como constructos puramente sociales y discursivos se está primando lo humano, como esencialmente constitutivo de las mismas, y relegando a los artefactos materiales científicos y tecnológicos.

Contra esta discriminación han surgido, en los estudios más recientes de ciencia y tecnología, replanteamientos críticos que se pueden también calificar de giro tecnológico.84 Este giro se manifiesta en una cierta ruptura con la sociología del conocimiento cientffico (SCC) animando a keliminar la primera $C$ de SCC, puesto que el tema central no es el conocimiento sino la práctica, y tambien la $S$, ya que no parece estar justificado atribuir una prioridad causal a lo social para comprender la práctica y la cultura científicasm. ${ }^{85}$

El interés central del estudio de la ciencia se ha reorientado claramente hacia la dimensión tecnológica de la misma:

Traditional accounts of science take it for granted that the end of science is to produce representations of how the world really is; in contrast, admitting a role for material agency points to the fact that, in common with technology, science can also be seen as a realm of instruments, devices, machines, and substances that act, perform, and do things in the material world. ${ }^{86}$

En esta línea, Andrew Pickering quiere "comprender la ciencia como un campo de dispositivos materiales operativos (y comprender las representaciones científicas en relación con estos dispositivos, antes que en sù uștal aislamiento esplendoroso) m. ${ }^{.77}$ Su propuesta es un análisis posthumanista de la practica cientffica que tenga realmente en cuenta el carácter tecnológico de la misma, reconociendo el papel de los agentes no-humanos (esto es, materiales) en la ciencia y su entramado con los agentes humanos. ${ }^{88}$

Bruno Latour ha sido uno de los primeros en abogar por una revisión de la SCC en esa misma dirección. Sus estudios se han centrado, desde un principio, en la práctica de los laboratorios como centros de la producción tecnocientífica. Para Latour, la ciencia y la tecnologia han de entenderse constructivamente, pero no están construidas socialmente, sy sino por entramados (collectives) de humanos y no humanos. "La obstinada devoción por la "construcción social" como recurso explicativon, en sociología del conocimiento científico como en sociología de la tecnologia, ha mantenido la dicotomía entre actores sociales y objetos, entre humanos y no humanos, cuando en realidad...

If anything, the modern collective is that in which the relations of human and nonhuman are so intimate, the transactions so many, the mediations so convoluted, that there is no plausible sense in which artifact, corporate body, and subject can be distinguished. $^{\text {9t }}$

En opinión de Latour, la filosofia de la tecnología tampoco ha ido muy le- 
jos, porque ha desconocido la producción conjunta de actores $y$ artefactos. ${ }^{y z}$ Nosotros podríamos añadir, debido a sus prejuicios humanistas y epistemológicos. La misma división entre filosofra de la ciencia y filosofía de la tecnología corre paralela a la separación de humanos y no humanos. Solo representa una división territorial acadêmica de entramados que nunca han estado separados en la práctica. Creo que, en la actualidad, está bastante claro que la filosofía de la ciencia no tiene sentido sin la filosofía de la tecnologia, ni a la inversa. A no ser que nos refiramos al sentido de los intereses territoriales.
En todo caso, si la filosofía de la ciencia y la filosofía de la tecnología han de tener un futuro que no sea pasar a formar parte del ya bien surtido museo filosófico, habrán de superar los viejos prejuicios tanto humanistas como epistemológicos, para integrarse interdisciplinariamente en los actuales estudios de ciencia y tecnologia. Mientras tanto, habría que empezar por integrar ambas ${ }^{93}$ en una filosofia postepistemológica y posthumanista de la tecnociencia, de acuerdo con el principio de que no debe separarse acadenicamente lo que viene unido en la practica y en la cultura. Pero esto es ya tema para otro trabajo.

\section{NOTAS}

1. Paul Edwards (ed.), The Encyclopedia of Philosophy, 8 vols., Nueva York, MacMillan, 1972.

2. Al establecer la contemplación (etopía) como el fundamento del conocimiento se instaura la división te6rica sujeto/objeto y la práctica queda epistemologicamente descalificada.

3. Cf. Manuel Medina, "Mito de la teoria y filosofia de la tecnología», Anthropos, $94-95$ (1989), pp. 35-39,

4. Cf. Mitcham, 1994, p. 119.

5. Ibid, p. 120.

6. ASobre las visiones platónicas en las que la viriud característica de la clase dominante es el conocimiento teórico, se levantan el primado político de la teoría y la descalificación politica de las technai. La sabidurfa politica se proyecta en las regiones de un conocimiento superior inalcanzable para artesanos y comenciantes - obligados a trabajar con sus propias manos para poder vivir-y solamente accesible para el reducido grupo que distruta del ocio y del placer. De la in. compatibilidad entre el conocimiento teŕrico y el saber técnico, se hace derivar la incompatibilidad entre la función política y la función tecnica. Para Aristoteles, la pertenencia al dominio de las técnicas productivas desealifica para la participacion en el dominio político. La deliberación politica es un asunto del discurso teórico para el que los artesanos están, tanto epistemologica como práticamente, incapacitados, En el refinado sistema teórico de Aristóteles la descalificacion cpistemológica conduce a la incapacidad etica y politica* (Manuel Medina, "La filosofia de la tecno. cracia», en Manuel Medina y José Sanmartín [eds.], Ciencia, Tecnologta y Soctedad. Estudios interäisciplinares en la universidad, en la educación y en la gestion publica, Barcelona, Anthropos, 1990, pp, 153-167).

7. Plutarch's Lives V, Londres, Heinemann, 1968, p. 472 s. (traducción del autor).

8. Ibid., p. $478 \mathrm{~s}$.

9. Ibid.s p. $470 \mathrm{~s}$.

10. José Ortega y Gasset, Meditación de la técntca, Madrid, Espasa-Calpe, 1965, p. 13.

11. Martin Heidegger, «Die Frage nach der Technik», en Vorträge urtd Aufsätze, Pfullingen, Neske, 1954, p. 21 (traducción del autor).

12. "El desocultamiento que impera en la técnica modema es un provocar que le exige a la raturalcza stministrar energia que como tal pueda ser extraída y almacenada. [...] Esta [provocación] se tealiza en tanto que se extrae la energia oculta en la naturaleza, lo extraído se transforma, lo transformado se almacena, lo almacenado a st vez se distribuye y lo distribuido se conmuta de nuevo. Extraer, transformar, almacenar, distribuir y conmutar son formas de desocultary (op. cit, p. 24).

13. Tbid, p. 31.

14. Entre otras muchas obras de Hugo Dingler, ct. Der Glaube und die Welmaschine und seine Ubenvindung, Stuttgart, Ferdinand Enke, 1932, y Uber die Geschichte und das Wesen des Experimentes, Munich, Eidos, 1952.

15. Op. cit., p. 40 .

16. Ibid, p. 36 .

17. Cf., p. ej., Friedrich Rapp, Analitsche Technikphilosophie, Friburgo, Karl Alber, 1978; Jean- 
Ives Goffi, La philosophic de la technique, Paris, PUF, 1988, y Don Ihde, Philosophy of Technology. An Introduction, Nueva York, Paragon House, 1993.

18. Esta corriente está representada principalmonte por Don Ihde, Michael Zimmermann y Albert Borgmann.

19. En la línea de Dewey destacan Paul Durbin y Lary Hickman. El autor más conocido en el campo de la filosofia política de la tecnologia es, sin duda, Langdon Wunner.

20. Jean-Ives Goffi, op. cit., p. 95 .

21. Jacques Ellul, *The Technological Onder", en C. Mitcham y R. Mackey (eds.), Philosophy and Technology: Readings in the Phitosophical Problenss of Technology, Nueva York, Free Press, 1972, p. 90 .

22. Ibid.

23. Cf. Mitcham, op. cit., p. 60.

24. La filosofía ahumanistica de la tecnología de Mitcham no coincide exactamente en la caracterización ni menos aún en la valoración con la que aquf se denomina filosofia humanista.

25. Mitcham, op. cit., p. 39 (traduccion del au. tor).

26. Ibid., p. 152.

27. Ibid. p. 39 .

28. bid.

29. Ibid, p. 113 .

30. Ibial.

31. Ibid., p. 64 .

32. Ibid, p. 39 .

33. La filosofía humanista es para Mitcham el "caté catén de las filosofias de la tecriologia por estat necesariamente "más cargada de filosofía $y$ sofisticación filosofica que la filosoffa ingeniertil de la tecnología, dado que la filosofía es una de las humanidades tradicionales y la ingeniería no lo esp (op. cit., p. 138).

34. Friedrich Rapp, Filosofia analtica de la tecnica. Buenos Aires, Alfa, 1981, p. 14.

35. lbid., p. 27.

36. Ibid, p. 60 .

37. Asociación de Ingenieros Alemanes.

38. La Engrneering Philosophy of Technolosy de Mitcham se identifica, practicamente, con la filosofía alemana de la tecnologia. La contraposicion de ésta con la Humanities Philosophy of Technology equivale a una confrontación nacional entre las filosofias norteamericana y alemana de la tecnologia.

39. Op. cit., p. 60 .

40. Ibid.

41. Bunge ha abogado desde el principio por la instituciondización de la filosofia de la tecnología y ha contribuido a la misma, pero siempre en clara oposicion a la corriente humanista. Algunos miembros de la SPT, como Joseph Pitt y Kristin Shrader-Frechette estarian cercanos a sus posiciones analíticas. En España, Miguel Angel
Quintanilla es quien ha desarrollado de forma más coherente y sistematica los planteamientos bungianos. Cf. su Techologfa: Un enfoque filosofico, Madrid, Fundesco, 1989.

42. Mario Bunge, aFive Buds of Techno-Philosophy", Technology in Society, 1 (1979), pp. 67-74.

43. Technology as Applied Sciencen es precisamente el título del artículo publicado en Tech. nology and Culture en 1966 que reproduce la contribución de Bunge al primer simposio sobre filosoffa de la tecnología (cf. supra).

44. Mario Bunge, La investigacion cientifica, Barcelona, Ariel, 1969, p. 43.

45. Ibid., p. 695.

46. Ibid, p. 694 .

47. Ibid.

48. Ibid., p. 695.

49. Asti p. ej.. el enunciado «Si se calienta un cuerpo imantado por encima de su punto de $\mathrm{Ct}$ rie, entonces pierde su imantación * sería el enunciado nomopragnático correspondiente a la regla tecnológica aPara desimantar un cuerpo, caliéntesele por encima de su punto de Curien. A su vez, dicho enunciado se derivaria de la ley cientifica si la temperatura de un cuerpo imantado rebasa su punto de Curie, entonces el cuerpo pierde su imantacion.

50. Bunge, op. cit., p. 699.

51. Ibid., p. 696.

52. Ibid., p. 699 .

53. Ibid, p. $695 \mathrm{~s}$.

54. Ibid, p, 683 .

55. Ibid., p. 684

56. Bunge se ha referido a los escritos de Heiddeger y Ellul sobre la tecnica diciendo que no son filosofía sino mala literatura (Bunge, «Five Buds of Techno-Philosophy», p. 68). Mitcham, más moderado, afirma que la filosofia analitica es incapaz de reconocer las propias limitaciones (Mitcham, op. cit., p. 140).

57. «Like all previous philosophy, the philosophy of technology raises in a new form perennial questions that are not subject to any straightforward resolution (ibid, p. 113).

58. Ibid., p. 153.

59. Ibid., p. 156 .

60. «Philosophy is not science, nor is it technology" (ibid, p. 113).

61. Ibid., p. 156.

62. En 1976 tuvo lugar un simposio de la $\mathrm{Fhi}$. losoplyy Association litulado "¿Hay alguna cuestión filosoficamente interesante en la tecnologla?». Ronald Giere se encargó de exponer los resultados. Ilegando a la conclusión de que la filosofia de la tecnologia no debfa considerarse camo otra especialidad dentro de la filosoffa, sino como una wilosofia aplicada. fef. Carl Mitcham, eQue es la filos ofia de la techologia?, Barcelona, Anthropos, 1989 , p. 189 s.). De hecho, los practicantes de la filosofía analítica de la ciencia no han sabi. 
do, en general, por dánde empezar con la tecnologla. Hace algunos ẫios, cuando la filosoffa de la tecnologla empezaba a conocerse en España, un colega del area de logica y filosolía de la ciencia me argumentó que la filosofia de la tecnologfa era imposible, pues ano se puede hacer filosofia de una camara fotograficas.

63. La predilección de Bunge se orientaría obviamente hacia la ciencia, mientras que la de Mitcham seria hacia la filosoffa tradicional.

64. Hickman, 1990.

65. Thid., p. 115.

66. Ibid., p, 46.

67. Ibid., p. 116.

68. Forman parte de la misma, entre otros, Pe. ter Janich, Jürgen Mittelstiass, Kuno Lorenz, Christian Thiel, Holm Telens y Rüdiger Inhetveen.

69. Lorenzen, 1978, p. 153 (traduccion del autor).

70. Lorenzen, 1987, p. 18.

71. Lorenzen, 1986, p. 23.

72. Ibid., p. 24.

73. Cf. Janich, 1978; Tetens, 1987.

74. Cf. Peter Janich, 1978.

75. Cf. Peter Janich, 1988. La filosofia constructiva de la ciencia como tecnología aplicada tiene obviamente radicales consecuencias para la politica de la ciencia y la tecnología, asi como para las relaciones entre ciencia, tecnologia y poIftica. En terminos de Lorenzen, «Toda teoría solo puede comprenderse sobre la base de una practica: este es el primado de la práctica. [...] toda práctica técnica presupone, al menos, formas previas de unä práctica política. Junto con el primado de la práctica respecto a la teoria vale por tanto, además, el primado de la política respecto a la técnicas (Lorenzen, 1978, p. 153). Véase en este contexto: Manuel Medina, «studios de ciencia y tecnologia para la evaluación de tecnologits y la política cientffica», en Jose Sanmartín et al. Superando fronteras. Estudios europeos de Ciencia-Tecnoloria-Sociedad y Evaluación de Tecnologias, Barcelona, Anthropos, 1994, pp. 95-126. José Sanmattín, «Ingeniería genética humana: evaluación y percepción públicas de las tecnologías genéticas», en José Sanmartín, Manuel Medina et al. Estudios sobre soctedad y tecnologia, Barcelona, Anthropos, 1992, pp. 224-265.

76. En Espana, Manuel Medina y Jose Sanmartín, vinculados personalmente a la comiente de la filosofín constructiva de la ciencia, han desarrollado sus planteamientos integrandolos en los actuales estudios de ciencia y tecnología.
Cf. Manuel Medina y José Sanmartín, -A New Role for Philosophy and Technology Studies in Spain, Technology in Society, 11 (1989), pp. 447. 455; :Filosofía de la Tecnologia. Una filosofía operativa de la tecnologia y de la ciencias, Anthropos, 94195 (1989); Manuel Medina, De la techne a la tecnotoria, Valencia, Tirant lo Blanch, 1985; José Sanmartín, Los nuevos redentores. Refexiones sobre la ingentieria genética, la sociobiolo. gia y el mundo feliz que ros pronzeten, Barcelona, Anthropos, 1987; Manuel Medina y Jose Sanmartin (eds.), Ciencia, Techologia y Sociedad. Estudios interdisciplinares en la universidad, en la educacion y en la gestión publica, Barcelona, Anthro pos, 1990; Jose Sanmartin, Tecnologia y futuro humaito, Barcelona, Anthropos, 1990.

77. Cf. p. ej., Steven Shapin y Simon Schaffer. Leviathan and the Air-Pump. Hobbes, Boyle and the Exporimental Life, Princeton, Princeton University Press, 1985; Peter Galison, How Experiments End, Chicago, The University of Chicago Press, 1987; David Gooding. Trevor Pinch y Simon Schaffer (eds.), The Uses of Experiment, SIt dies in the Natural Sciences, Cambridge, Cambrict ge University Press, 1989.

78. Price, 1984.

79. Hacking, 1983.

80. Op. cit., p. 167

81. Ibid., p. 181

82. Hacking, 1992, p. 30 (traducción del autor).

83. Es lo que Pickering llama reduccionisno sociologico (Pickering, 1992, p. 14 s.) y Latour sociologismo (Latour, 1994, p. 42).

84. Woolgar se ha referido tambión a un giro tecnologico en los estudios sociales de la ciencia, pero en un sentido distinto al que aquí se expone (Steve Woolgar, aThe Turn to Technology in Social Studies of Science, Science, Technology \& Hunan Values, 16, n." 1 [1991], pp. 20-50).

85. Pickering, 1992, p. 14 (traduccion del autor).

86. Pickering, 1993, p. 563.

87. Pickering, ibid. La consonancia con la filosofía constructiva de la ciencia es asombrosa.

88. Ibid, pp, 562 y 567.

89. Latour, 1994, p. 53.

90. Ibid., p. 54 (traducción del autor).

91. Ibid., p. 53.

92. Ibid., p. 30 ss.

93. Don Ihde, 1991, apunta también hacia una cierta integración de la fílasoffa de la ciencia y la filosofía de la tecnología, aunque en un contexto fenomenológico. 


\title{
REFERENCIAS
}

HACKING, Ian (1983); Reprasenting and Intervening, Cambridge, Cambridge University Press.

- (1992): aThe Self-Vindication of the Laboratory Sciencess, en A. Pickering (ed.), Science as Practice atd Culute, Chicago, The University of Chicago Press, 29-64.

Hickman, Iany (1990): John Dewey's Pragnatic Tech tology, Bloomington, Indiana University Press.

IHDE, Don (1991): Instrumental Realism. The Interface between Philosophy of Science and Philosophy of Technology, Bloomington, Indiana University Press.

JANich, P. (1978): "Physics - Natural Science or Technology?*, en W. Krohn, E. Layton $y$ P. Weingart (eds.). The Dynamics of Science and Technology, Dordrecht, Reidel, 3-27.

- (1988): :Truth as Success of Action. The Constructive Approach in the Philosophy of Sciencex, en K. Hronszky, M. Feher y B. Dajka (eds.). Scientific Knowledge Socialized, Dordrecht, Kluver, 313-326.

LAtour, Buno (1994): «On Technical Medition Philosophy, Sociology, Genealogym, Conmon Knowledge, 29-63.

LORENZEN, Paul (1978): Theorie der technischen und politischen Vernunf, Stutgart, Reclam.
- (1986): «Das technische Fundament der Geometrie; en $C$. Bumichter, $R$. Inhetveen y $R$. Kotter (eds.). Techntische Rationalität und rationale Heuristik, Munich, Schoningh, 17-25.

- (1987): Lehrbuch der konstruktiven! Wissenschaftsheorie, Mannheim, Wissenschaftsverlag.

MiтchaM, C. (1994); Thinking through Technology. The Path between Engineering and Philosophy, Chicago, The University of Chicago Press.

Prckerunc, A. (1992): «From Scjence as Knowledge to Science as Practicex, en A. Pickering (cd.), Science as Practice and Culture, Chicago, The University of Chicago Press, 1-26.

- (1993): «The Mangle of Practice: Agency and Emergence in the Sociology of Science, Anterican Jountal of Sociology, 99, n." 3, 559-589.

Puce, Derek Desolla (1984): "The Seience/Technology Relationship, the Craft of Experimental Science, and Policy for Improverment of High Technology Innovation, Research Policy, $13,3-20$

TETENS, Holm (1987): Experinentelle Erfahruny. Eine wisseschafstheoretische Studie iber die Rolle des Experiments in der Begriffs- und Theoriebilany der Physik, Hamburgo, Felix Meiner.

\section{La sociología y la naturaleza social de la ciencia}

\author{
JESÚS SÁNCHEZ NAVARRO \\ Universidad de La Laguna
}

En 1928, en pleno auge del Positivismo Lógico, Carnap propuso un programa de trabajo que tuvo importantes consecuencias posteriores. Según Carnap la Epistemología debía realizar una reconstrucción racional de los procesos de conocimiento y 'conformación de la realidad' que en la mayoría de los casos llevamos a cabo intuitivamente. Consideraba tal reconstrucción descriptiva, fidedigna y siguiendo ula forma racional de derivaciones lógicas». Llevarla a cabo con los conceptos de todos los campos del conocimiento, incluyendo la ciencia, era desde su punto de vista el problema fundamental de la filosotia y la epistemología."

Diez años después y aplicando la propuesta de Carnap a la Filosofía de la Ciencia, Reichenbach establecia dos distinciones que ya habian sido insinuadas por otros autores y que hicieron fortuna rápidamente. ${ }^{2}$ La primera era la diferencia entre las relaciones internas y externas del conocimiento. Llamaba 'internas' a las que 